\title{
Cuidados del personal de enfermería en la dimensión espiritual del paciente. Revisión sistemática
}

\section{Care nursing the spiritual dimension of the patient.} Systematic review

\section{Cuidados equipe de enfermagem a dimensão espiritual do paciente. Revisão sistemática}

\author{
María Teresa Pinedo Velázquez ${ }^{1}$ Juan Carlos Jiménez Jiménez² \\ ${ }^{1}$ Doctorada en Enfermería Universidad de Alicante. Profesora Enfermería Universidad CEU San Pablo- Elche. \\ ${ }^{2}$ Grado Enfermería. Hospital Universitario Vinalopó Salud. \\ Cómo citar este artículo en edición digital: Pinedo Vázquez, M.T., \& Jiménez Jiménez, J.C. (2017). Cuidados del personal de \\ enfermería en la dimensión espiritual del paciente. Revisión sistemática. Cultura de los Cuidados (Edición digital), 21(48). \\ Recuperado de < http://dx.doi.org/10.14198/cuid.2017.48.13> \\ Correspondencia: \\ Correo electrónico: tpinedo.el@uch.ceu.es \\ Recibido: 20/12//2016; Aceptado: 10/03/2017
}

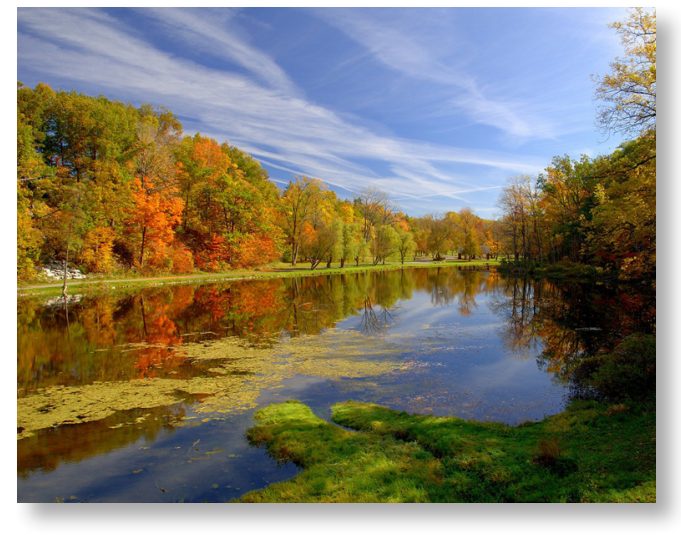

\section{ABSTRACT}

It is necessary to know the perception of nurses about the emotional attention given to patients at the spiritual dimension in patient care plan. The objective was to know the spiritual care offered by nurses in their daily work during a patient's hospital stay and detect possible associated factors that hinder the facing death.

Material and Methods. The unit of analysis has been the primary original studies, electronic searches (Cuiden, Scielo, Pubmed) and manuals relevant of articles published in journals, bibliographies and reference lists of reviews and papers were made.

Results and Conclusions. There are a number of factors that hinder the proper intervention of nursing professionals in specific situations of non-health: especially when the patient feels the urgent need to obtain information on their health status because it is admitted in a hospital in terminal situation, as when you communicate suddenly having a serious organic disorder in some body structure with some degree of limitation prevents regular physical activity.... as a result he feels emotions like anxiety, fear, depression, anger, dissatisfaction, etc which are emotions with high emotional and spiritual burden.

Keywords: Palliative Care, spirituality, nursing

\section{RESUMEN}

Se precisa conocer la percepción de los profesionales de enfermería acerca de la atención emocional y espiritual prestada en el plan de cuidados del paciente. El objetivo fue conocer la atención espiritual que brindan las enfermeras y enfermeros en su trabajo diario durante el pro- 
ceso de estancia hospitalaria de un paciente así como detectar posibles factores asociados que afloran al enfrentarse a la muerte.

Material y Métodos. La unidad de análisis son los estudios originales primarios. Se hicieron búsquedas electrónicas (Cuiden, Scielo, Pubmed) y manuales de artículos relevantes recientemente publicados en revistas, bibliografías y listas de referencias de revisiones y documentos.

Resultados y Conclusiones. Existen una serie de factores que dificultan la adecuada intervención del profesional de enfermería en situaciones concretas de no salud: principalmente cuando el paciente siente la necesidad imperiosa de obtener información sobre su estado de salud porque se encuentra ingresado en un centro hospitalario en situaciones terminales, así como también cuando le comunican de repente que tiene un grave trastorno orgánico en alguna estructura corporal y con algún grado de limitación que le impide una actividad física habitual de repente... como consecuencia de ello se enfrenta a emociones como la angustia, el miedo, la depresión, la rabia, la insatisfacción, etc que son emociones con alta carga emocional y espiritual.

Palabras clave: Cuidados paliativos, espiritualidad, enfermería

\section{RESUMO}

É necessário conhecer a percepção dos enfermeiros sobre o cuidado emocional e espiritual previsto no plano de cuidados do paciente. O objetivo foi conhecer a assistência espiritual oferecida por enfermeiros no seu trabalho diário durante a internação do paciente e detectar possíveis fatores associados que surgem quando enfrentar a morte.

Material e Métodos. A unidade de análise é os estudos originais primários. buscas eletrô- nicas (CUIDEN, SciELO, PubMed) e manuais de artigos relevantes publicados recentemente em revistas, bibliografias e listas de referência de comentários e papéis foram feitas.

Resultados e conclusões. Há uma série de fatores que dificultam a intervenção adequada dos profissionais de enfermagem em situações específicas de não-saúde: especialmente quando o paciente sente-se a necessidade urgente de obter informações sobre o seu estado de saúde, porque é admitido em um hospital em situações terminais, como quando você se comunica de repente tem uma doença orgânica grave em alguma estrutura do corpo com algum grau de limitação impede que a atividade física regular, de repente ... como resultado enfrenta emoções, como ansiedade, medo, depressão, raiva, insatisfação, etc, que são emoções com alta carga emocional e espiritual.

Palavras-chave: Cuidados Paliativos, espiritualidade, enfermagem.

\section{INTRODUCCIÓN}

La muerte es un fenómeno natural que forma parte del ciclo vital de todos los seres vivos Enfermería juega un papel relevante, cuando en su trabajo tiene que enfrentarse a la muerte, planteando la necesidad de saber cómo actuar en estas situaciones que generan gran sufrimiento y ansiedad tanto para la persona que vive el proceso de final de su vida, así como para los profesionales, condicionando su manera de proporcionar unos cuidados de calidad ajustado a las necesidades del paciente para ayudarles a tener una buena muerte (Ortega, 2013)

En los últimos años la expansión de los cuidados paliativos, ha fomentado el desarrollo de modelos de evaluación y acompañamiento espiritual, que según van adquiriendo 
reconocimiento académico, facilitan la integración de este ámbito en la práctica clínica. Esto equivale a tener mapas del territorio por el que transitan nuestros pacientes y que ayudan a orientar a los profesionales a asumir su acompañamiento (Sierra, 2015).En este contexto resulta cada vez más necesario ofrecer ayudas de todo tipo cuando el ser humano enferma y sufre ya que además de los dolores del cuerpo están los que se pueden llamar dolores del alma: soledad, miedo, tristeza, temo, etc. En nuestro entorno, la atención espiritual puede atribuirse a la valoración de la esfera emocional (valores y creencias) siendo difícil su evaluación y abordaje desde el paradigma científico enfermero. Actualmente se reconoce la atención espiritual como procedimiento a seguir dentro de unos estándares de calidad asistencial del Ministerio de Sanidad, Política Social e Igualdad donde vienen definidas las líneas de actuación mediante un protocolo existente en la guía de Cuidados Paliativos dentro del sistema nacional de salud. Las estrategias de actuación contemplan varios niveles de intervención, básico, intermedio y avanzado. Estos equipos están formados por enfermeras, médicos, psicólogos, auxiliares de enfermería y trabajadores sociales, además de fisioterapeutas o sacerdotes en función de la evolución de la enfermedad (Benito,2016).

\section{MARCO TEÓRICO}

Remontándonos a la antigua Grecia el filósofo Platón ya habló del ser humano como ser dual compuesto de cuerpo y alma entendiendo esta como espíritu, donde con la muerte del cuerpo el alma se liberaba, permaneciendo así lo esencial del hombre, su parte espiritual (Morillo, 2013). El constructo espiritualidad es algo subjetivo y multidimensional donde cabe la interpretación que cada persona quiera dar adecuado a su modo de entender la vida, esto hace que sea complicado su estudio (Ortega, 2013) Es precisamente su subjetividad lo que hace que la espiritualidad se confunda en múltiples ocasiones con la religiosidad teniendo ambos conceptos puntos en común sin ser coincidentes Desde las ciencias enfermeras son muchas las teóricas que han contemplado la salud desde una perspectiva más amplia y holística, asumiendo la interacción de múltiples sistemas que convierten a la persona en mucho más que en la suma de sus partes. Desde el nacimiento de la enfermería son muchas teóricas que asumen como parte inherente a la enfermería, tener una visión global del cuidado donde necesariamente se aborde al ser humano como ser espiritual. Florence Nightingale afirmó que es parte intrínseca del ser humano su dimensión espiritual y por tanto la necesidad de su cuidado. Marcos teóricos como el de Watson, Ray o Kolcaba incorporan la dimensión espiritual tanto a la salud como al cuidado, en contraposición al modelo biomédico estando la literatura de enfermería más desarrollada en este área que otras disciplinas. Espiritualidad y religión están relacionadas sin ser coincidentes. El aspecto religioso está relacionado con la fe en un ser o poder superior, mientras que la espiritualidad es entendida como un fenómeno relacionado con el sentido de la vida y la transcendencia (Galiana, 2014)Estos estudios han puesto de manifiesto que la espiritualidad es una necesidad del paciente, siendo los constructos más frecuentes a los que se hacen referencia: la necesidad de esperanza, necesidad de sentido, necesidad de amor, necesidad religiosa o divina y necesidad de afrontamiento de la muerte(Payás, 2015)

Así mismo Virginia Henderson plantea cuidados desde un enfoque holístico en el que se engloban aspectos biológicos, sociales, psi- 
cológicos, culturales y espirituales. Desde el punto de vista de Watson una vida espiritual rica tiene un impacto positivo en la salud física y psicológica del paciente. Madeleine Leininger definió la base de los cuidados de enfermería desde la cultura y la espiritualidad (Pinedo, 2009). Los cuidados paliativos en España tuvieron su desarrollo en los años 80, así en 1987 se fundó la primera Unidad de Cuidados $\mathrm{Pa}$ liativos en el Hospital de la Santa Cruz de Vic (Montes de Oca, 2006). En 1990 la OMS adopta la definición propuesta por la EAPC de los cuidados paliativos como "el cuidado total y activo de aquellos pacientes cuya enfermedad no responde a un tratamiento curativo, siendo primordial el control de síntomas y de los problemas psicológicos, sociales y espirituales" dando como resultado un modelo de atención en cuidados paliativos. Desde cuidados paliativos el abordaje de la dimensión espiritual lleva asociadas ciertas dificultades en la práctica clínica para acercarnos a la persona que sufre, para reconocer, atender y acompañar en este proceso (Sierra, 2015). está considerada condición indispensable para un correcto cuidado integral del paciente en cuidados paliativos, cuando la enfermedad aparece como incurable y la persona se enfrenta con su problema terminal, los profesionales que acompañan este proceso necesitan actitudes y herramientas para facilitar en la medida de lo posible serenidad y confianza, en este sentido el acompañamiento espiritual merece ser incorporado como parte integral de los cuidados paliativos (Moraes, 2015)

\section{EJECUCIÓN DE BÚSQUEDA DE LITERA-} TURA

Para la búsqueda de los artículos se han revisado diferentes fuentes bibliográficas y bases de datos. Se ha realizado una búsqueda biblio- gráfica desde 01 de septiembre del 2015 hasta 31 de enero de 2016. Los criterios de inclusión utilizados para la selección de los artículos han sido artículos incluidos en el período 2005-2016, con descriptores específicos. Los criterios de exclusión contemplaron aquellos artículos que no cumplían esos criterios de inclusión. Las bases de datos utilizadas han sido:

CUIDEN: Se usaron los siguientes descriptores: Cuidados Paliativos, Espiritualidad y Enfermería, obteniendo 18 resultados de los que se revisaron 12 artículos y de estos se seleccionaron para el estudio 4 artículos descargados directamente de la base de datos.

SCIELO: Se utilizaron los siguientes descriptores: Cuidados Paliativos, Espiritualidad y Enfermería, obteniendo 52 resultados de los que se revisaron 10 artículos y se descargaron 5 artículos directamente de la base de datos.

PUBMED: Se usaron los siguientes descriptores: Cuidados Paliativos, Espiritualidad y Enfermería, obteniendo 681 resultados de los que se revisaron 23 artículos y se descargaron 2 artículos directamente de la base de datos.

\section{RESULTADOS}

\section{Categoría 1: Importancia de la espirituali- dad en clínica.}

Se observó que en casi la totalidad de los documentos analizados se evidencia la importancia de atender esta dimensión del ser humano como parte fundamental en el proceso final de la vida como garantía de un bienestar espiritual, cuanto más si cabe en cuidados paliativos. En el estudio de Bermejo (2012) donde el objetivo del estudio fue evaluar cómo percibían los pacientes y cuidadores principales la atención espiritual en una unidad de cuidados paliativos: un 92,5\% de los pacientes veía necesaria la atención espiritual durante la 


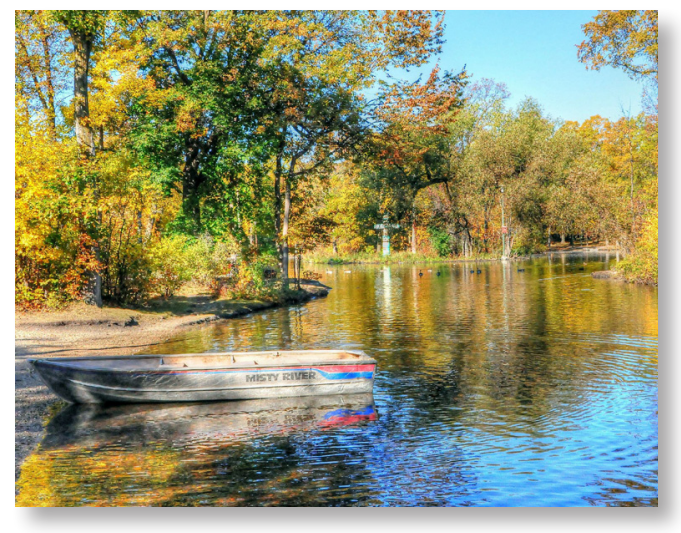

enfermedad. Del mismo modo en estudio Balboni (2013) donde el objetivo era determinar qué factores influían en el escaso cuidado espiritual por parte de enfermeros y psicólogos a enfermos al final de la vida, se concluyó unos efectos beneficiosos con los cuidados espirituales, manifestados tanto por pacientes como por el equipo sanitario. Se reconocía como limitación del estudio un sesgo en la población por centrarse el estudio únicamente en una región de Estados Unidos.

Estos resultados están apoyados por otro estudio de Delgado (2011) donde el objetivo fue examinar la prevalencia e intensidad de la espiritualidad, de cómo el dolor espiritual estaba asociado a unos síntomas y con una mejor calidad de vida y donde los pacientes daban una importancia a la atención espiritual y religiosidad (98\%) también reconocieron los pacientes que el dolor espiritual agravaba sus síntomas físicos y emocionales por lo que afirmaron que la atención espiritual y religiosa les servía como fortaleza y confort para lidiar con su enfermedad oncológica.. Por otro lado Pinedo (2009) afirma que la espiritualidad y la religión son fortalezas que ayuda a la persona a encontrar significado, esperanza, alivio y paz interior, concluyendo diciendo que la evidencia dice que la espiritualidad es universal y que su práctica está en relación con una mejor sa- lud mental, una mejor salud física y una mejor adaptación a la enfermedad. Sin embargo en el estudio de Moraes (2015) cuyo objetivo del estudio es analizar las percepciones de enfermeras sobre el cuidado espiritual, afirma que ve como necesaria la atención espiritual pero prevaleciendo siempre el aspecto físico de los pacientes.

\section{Categoría 2: Ambigüedad del concepto de espiritualidad.}

El análisis de los documentos seleccionados evidencia la subjetividad del concepto de espiritualidad en su interpretación por cada uno de los profesionales, siendo un gran obstáculo para el reconocimiento de las necesidades de carácter espiritual que el paciente pueda tener y para la posterior intervención en un cuidado integral. Sandoval (2014) en su estudio cuyo objetivo fue conocer la concepción de espiritualidad y religiosidad de un equipo multidisciplinar de cuidados paliativos oncológicos observó como el valor dado al concepto "religiosidad" era más alto que a la palabra "espiritualidad". Los pacientes definieron la espiritualidad con palabras como: paz, fe, Dios, creencia, alma, amor, tranquilidad, religión, armonía, esperanza, meditación, bondad, espíritu, transcendencia y creencias. Del mismo modo definieron religiosidad con palabras como: fe, Dios, iglesia, esperanza, creencia, ritos, religión, amor, fanatismo, espiritualidad, compromiso, oración, reglas y sacerdote.

El estudio de Uribe y Lagoueyte (2014) donde el objetivo era conocer el significado que tenía el cuidado espiritual para los enfermeros, se observó que los conceptos de religión y espiritualidad presentaban diversos significados, iban desde algo cultural a una opción personal, y por consiguiente resultaba complicado diferenciar ambos conceptos. En su es- 
tudio Terceño (2015) distinguió dos maneras de abordar los cuidados espirituales por parte de enfermería, una estaba relacionada con la religión y la otra que tenía que ver con el conocimiento interno de la persona, concluyendo que aunque sean maneras diferentes de entender los cuidados ambas eran perfectamente compatibles. Castanheira (2015) describía la comprensión del significado de la espiritualidad y la religiosidad de los enfermeros diciendo que resulta complicado para la enfermería expresar ambos conceptos. La enfermería considera la espiritualidad como algo subjetivo e intrínseco al ser humano, ven importante la percepción individual de la espiritualidad para dar un cuidado diferenciado. Por otro lado asocian la religión a grupos sociales con los mismos valores y opiniones sobre doctrinas o superioridades religiosas. Dones (2013) describía y analizaba el acompañamiento espiritual en cuidados paliativos evaluando posibles áreas de mejora, observó que al analizar si los profesionales no distinguían los aspectos religiosos de los espirituales, siendo evidente la necesidad de formación en este sentido.

\section{Categoría 3: Falta de formación de los pro- fesionales}

La falta de formación también impide un buen abordaje de la espiritualidad. Se evidencia como una preparación inadecuada afecta de dos maneras diferentes a los profesionales de enfermería que atienden a pacientes paliativos, por un lado repercute en su propio bienestar por el trato continuo con la muerte generando sentimientos de angustia, miedo y sufrimiento, y por otro lado condiciona los cuidados del paciente. Guedes y Oliveira(2013) se preocuparon por las las percepciones de las enfermeras que cuidan a personas en el proceso de morir, observando que la enfermera establece un vínculo afectivo con paciente y familia relacionado con el tiempo de interacción de la enfermera con estas personas lo que finalmente produce en la enfermera sufrimiento ante la muerte de los pacientes, viendo la necesidad de formarse de manera específica para dar importancia a la humanización del cuidado

Magalhaes (2014)con el objetivo de conocer la opinión de los estudiantes de enfermería sobre los cuidados de la dimensión espiritual, afirma que la falta de atención espiritual por parte de los estudiantes de enfermería se debe a su falta de preparación académica, generando cierta dificultad a la hora de aplicar cuidados espirituales.

Mesquita( 2014) en su trabajo sobre el bienestar del equipo de enfermería y la prestación del cuidado espiritual observó como el 77\% de los enfermeros no se sienten preparados para la prestación de cuidados espirituales, afirmando el $42 \%$ de los profesionales no haber recibido formación especifica al respecto. Payás (2015) mediante la obtención de datos empíricos sobre detección y atención de las necesidades espirituales de pacientes en cuidados paliativos, a través de un análisis cuantitativo de los datos, observó que el $31 \%$ de los participantes manifestaban tener dificultad a la hora de identificar necesidades espirituales, que el $41 \%$ afirmaba que pocas veces hablaban de la muerte con sus pacientes, que un $49 \%$ insistían que pocas veces se hablaba de necesidades espirituales en las sesiones clínicas realizadas en los servicios, e incluso que un $48 \%$ nunca había oído a sus pacientes expresar necesidades espirituales, porque no preguntaban por ello. A continuación con un análisis cualitativo se clasificaron las expresiones en tres dominios: interpersonal con un $24 \%$ de expresiones recogidas, intrapersonal con un $31 \%$ de expresiones y 
transpersonal con un $45 \%$ de respuestas recogidas, siendo este dominio donde más expresiones se identificaron. Estos resultados fueron apoyados por el estudio de Ortega y Gonzalez (2013) cuyo objetivo es conocer la visión de los profesionales de enfermería acerca de la dimensión espiritual de las personas al final de la vida, se evidencia una dificultad para incorporar la atención espiritual en estos cuidados así como una limitación por parte de los enfermeros por la dificultad que supone cuidar todas las dimensiones de las personas que se enfrentan a la muerte, siendo clara la necesidad de una madurez personal e individual para realizar esta función. La SECPAL (Sociedad Española de Cuidados Paliativos) conoce la importancia de establecer unas directrices comunes de actuación en al ámbito de los cuidados paliativos y establece directrices para que el equipo multidisciplinario de profesionales tenga muchas habilidades en la valoración y atención de los problemas espirituales del paciente, además reconoce la necesidad de formación en este ámbito y presenta algunas iniciativas como éstas: trabajar con la dimensión interna del profesional, sacando lo mejor de la persona como ayuda a su curación sin inculcar mensajes como verdades absolutas y utilizando una técnica metodológica de ayuda con planteamientos universales que buscan herramientas efectivas para el aprendizaje (Benito, 2014).

\section{Categoría 4: Estandarizar herramientas.}

En orden a ofrecer una revisión de las herramientas de medida de la espiritualidad en cuidados paliativos, hay que decir que las herramientas para la evaluación espiritual de las que se disponen en la actualidad no están adaptadas al castellano y algunas fueron diseñadas para realizar investigaciones concretas, por consiguiente son deficientes para su uso en práctica clínica (Tosao, 2012).Se identifica como principal criterio a seguir para una adecuada intervención espiritual el uso de instrumentos estandarizados de medición específicos, y herramientas estructuradas de valoración práctica que faciliten una óptima intervención en las inquietudes religiosas, espirituales y existenciales de las personas. Galiana (2014) hace referencia a la necesidad del uso de herramientas estandarizadas por parte de los profesionales sanitarios, tras el análisis de 57 escalas de valoración de la espiritualidad, concluye identificando 29 escalas de las que tan solo una la Functional Assessment of Chronic Illness Therapy-Spiritual WellBeingScale (FACIT-sp) esta validada transculturalmente. Coincidiendo también en la falta de herramientas transculturales, encontramos a Rudilla (2015) cuyo estudio tiene como objetivo ofrecer evidencia sobre la eficacia del counselling para la mejora de la espiritualidad, donde se evaluá la espiritualidad antes y después de la intervención counselling, concluyendo los datos en un efecto positivo sobre la espiritualidad. Se proponen las actitudes psicológicas como empatía, autenticidad y aceptación incondicional, así como las actitudes espirituales como compasión, presencia y hospitalidad, como estrategias facilitadoras para poder aplicar los diferentes modelos de intervención. Braz (2016) ha tenido como objetivo comprender la perspectiva enfermera de la espiritualidad aplicada en cuidados paliativos; explica que las enfermeras usan como estrategia para detectar necesidades espirituales en los pacientes la comunicación, la escucha, la música ambiental, la colaboración con otros profesionales, etc destacando la comunicación verbal y la escucha como un elemento eficaz en los pacientes sin 
posibilidad de curación. Este estudio coincide con la SECPAL en su modelo de counselling espiritual como herramienta de intervención para el acompañamiento.

\section{Categoría 5: Modelo biomédico en la atención.}

La dimensión biomédica ha sido tratada por Pinedo (2009) Magalhaes( 2012) y Terceño (2015) la cual centra su atención en la dimensión biológica y física de las personas, no teniendo en cuenta como la persona pueda vivir y sentir la enfermedad, ni teniendo en cuenta como puede afectarle a su entorno familiar, enfatizando todos por igual la parcialidad del cuidado ofrecido por el enfoque biomédico el cual centra el cuidado en los problemas físicos.

\section{CONCLUSIONES}

Esta búsqueda ha permitido reflexionar acerca de los instrumentos utilizados para la medición del sufrimiento espiritual, concluyendo en que existe una necesidad de generar propuestas o estándares de cuidado que contribuyan a cualificar y registrar la práctica de la atención espiritual explorando algo tan sutil y subjetivo como es la atención emocional de la persona que pierde su salud y vivencia malestar y dolor espiritual. A partir de esta reflexión, hay que posicionarse en la falta de consenso sobre medidas de actuación protocolizadas, en consensuar el término "acompañamiento espiritual", y en aumentar la formación de los profesionales para que sepan reconocer estas necesidades en los pacientes que cuidan. Una primera recomendación sería incluir en la entrevista inicial de valoración de enfermería una serie de indicadores que detecten y aborden una situación de sufrimiento que el paciente vivencia: miedo, angustia, rabia, insatisfacción, soledad, tristeza y dolor interior.

\section{REFERENCIAS}

- Balboni, M., Sullivan A., Amobi A., Phelps, A., Gorman, D., Zollfrank, A., Peteet J., Prigerson, G.,Vanderweele, T., \& Balboni, A. (2013).Why is spiritual care infrequent at the end of life? Spiritual care perceptions among patients, nurses and physicians and the role of training. Journal of Clinical Oncology, 31(4), 461-467.

- Braz, C., Limeira, M., Geraldo, S., Da Silva, F., Souza, P., \& De Oliveira, R. (2016). Spirituality in patient care under palliative care: a study with nurses. Escola Anna Nery Revista de Enfermagem, 20 (1),176-182.

- Benito, E., Barbero, J., \& Dones, M. (2016). Monografías Sociedad española de Cuidados Paliativos (SECPAL) España. Recuperado en http:// www.secpal.com/\%5CDoc umentos $\% 5$ CBlog\% 5CMonografia\%20secpal.pdf

- Bermejo, J., Lozano, B., Villacieros, M., \& Carabias R. (2012). Opinión delos profesionales de cuidados paliativos sobre la atención espiritual. Ética de los Cuidados. 5(10). Recuperado en en: http://www.humanizar.es/fileadmin/ documentos/Investigacion/AtenciA3n_espiritual_en_ cuidados_paliativos_Valoracion_y_vivencia_de_los_ usuarios.pdf

- Bermejo, J., Lozano, B., Villacieros, M., \& Gil M. (2012). Atencion espiritual en cuidados paliativos. Valoración y vivencia de los usuarios. t, 47, 10. Recuperado en http://www.josecarlosbermejo.es/sites/ default/files/opinion_

de_los_profesionales_de_cuidados_paliativos_sobre_la_ atencion_espiritual_etica_de_los_cuidados.pdf

- Castanheira, L., Moreiras, T., Santos, F., Pan, R., Santos, M., \& Melo, S. (2013). Espiritualidade e religiosidade na perspectiva de enfermeiros. Texto Contexto Enferm 22(1), 52-60.

- Delgado, M., Hui, D., Parsons, H.,Govan, K., De la Cruz, M., Thorney, S., \& Bruera, E. (2011).

Spirituality, religiosity and spiritual pain advenced cáncer patients., Pain Symptom Manage 41(6), 986-994. - Dones, M., Collette, N., Barbero, J., Gomis, C., Balbuena, P., \& Benito, E. (2013). ¿Cómo percibimos los profesionales el acompañamiento espirituale Cuidados 
Galiana, L. et al (2014). C.E.I. espiritual en cuidados paliativos: una revisión crítica.21(2), 62-74.

- Guedes, E., \& De Oliveira D. (2013).Vivencia de las enfermeras ante los cuidados en el proceso de muerte. (2013). Index Enferm 22 (1-2). Recuperado en http://scielo.isciii.es/scielo.php?

pid=S1132-12962013000100006\&script=sci_arttext

- Magalhaes, A., Limeira, M., Braz, C., Lima, E., Geraldo, S., \& Pereira, A. (2012). The spiritual dimension of care in nursing practice: student's opinión. JNurs UFPE, 6 (9), 2037-2044.

- Mesquita, A., Costa, C., Barreto, M., Souza, F., y López

E. (2015). El bienestar espiritual y la prestación del cuidado en un equipo de enfermería. Index Enferm 23 (4). Disponible en: http://scielo.isciii.es/scielo. php?script=sci_arttext\&pid=S1132-12962014003300006

- Moraes, O., Andrade, G., Almeida, I., \& Dias M. (2015). Spiritual care in nursing practice: Nurses perception. Rev. J Nurs UFPE, 9 (8), 8817-23.

- Ramos, A., Morillo, A., Gayo, N., Tasiguano, J., Munzón, E., \& Ribeiro, A.(2013 Curar o paliar: ¿qué cuesta más?Análisis de costes del tratamiento de una herida crónica en función de su finalidad. Medicina Paliativa 22(2), 45-51.

- Ortega, A., \& González, M. (2013). El valor de la dimensión espiritual en el final de la vida desde la perspectiva de los profesionales de enfermería. Med Paliat, 134, 1-6.

- Payás, A., Barbero, J., Bayés, R., Benito, E., Giró, R., Maté, J., Rodeles, R., \& Tomas, C. (2015). ¿Cómo perciben los profesionales de paliativos las necesidades espirituales del paciente al final de la vida? Medicina Paliativa, 15 (4), 225. Recuperado en http://cat.inist.fr/? aModele $=$ afficheN\&cpsidt $=20578364$

- Pinedo, M.T. (2009). La humanización de los cuidados: Reto de la enfermería del siglo XXI. Haciendo visible, lo invisible. El papel de enfermería frente al sufrimiento espiritual del paciente. Valencia: Consejo de Enfermería de la Comunidad Valenciana.

- Rudilla, D., Oliver, A., Galiana, L., Barreto, P. (2015). Espiritualidad en atención paliativa: evidencias sobre la intervención con counselling. Psychosocial Intervention, 24 (2). Recuperado en http://scielo.isciii.es/pdf/inter/ v24n2/articulo3.pdf

- Sandoval, P., Rangel, N., Allende, S., \& Ascencio, L. (2014). Concepto de espiritualidad del equipo multidisciplinario de una unidad de cuidados paliativos: un estudio descriptivo. Rev. Psicooncología 11(2-3), 333344.

- Sierra, M., \& Getino, M. (2013). La atención espiritual al final de la vida en los domicilios. Rev Rol Enferm, 36 (9), 596-60.

- Tosao, C. (2012). Abordaje aconfesional de la espiritualidad en cuidados paliativos. Formación Médica continuada en Atención Primaria, 19 (6), 331-338.

- Terceño, C. (2010). Espiritualidad y cuidados de enfermería: una visión panorámica. Ética de los Cuidados, 3 (5). Recuperado en http://www.index-f.com/ eticuidado/n5/et7346r.php

- Uribe, S., \& Lagoueyte M. (2014). Significado del cuidado espiritual: la mirada de los profesionales de enfermería. Avances en Enfermería, 23 (2), 261-270. 